Instituto Internacional de Investigación y Desarrollo Tecnológico Educativo INDTEC, C.A.

DOI: https://doi.org/10.29394/Scientific.issn.2542-2987.2021.6.21.4.81-100

OAl-PMH: http://www.indteca.com/ojs/index.php/Revista Scientific/oai

Artículo Original / Original Article

\title{
Estrés Laboral y Salud General en Trabajadores Administrativos del área Bancaria
}

\author{
Autores: Darwin Raúl Noroña Salcedo \\ Universidad Regional Autónoma de Los Andes, UNIANDES \\ darwin norona@yahoo.com \\ Quito, Ecuador \\ https://orcid.org/0000-0002-0630-0456 \\ Valeria Alejandra Quilumba Vallejo \\ Universidad Regional Autónoma de Los Andes, UNIANDES \\ conejavqv@gmail.com \\ Ambato, Ecuador \\ https://orcid.org/0000-0001-9350-9735 \\ Vladimir Vega Falcón \\ Universidad Regional Autónoma de Los Andes, UNIANDES \\ vega.vladimir@gmail.com \\ Ambato, Ecuador \\ https://orcid.org/0000-0003-0140-4018
}

\section{Resumen}

En los diferentes puestos de trabajo los seres humanos se encuentran expuestos a factores de riesgo psicosociales, es ineludible y están condicionados con el puesto de trabajo. El aparecimiento del COVID-19 incrementó el efecto nocivo en la salud. El objetivo de la presente investigación fue determinar cómo el estrés laboral afecta la salud general del personal administrativo de la Cooperativa de Ahorro y Crédito, San José, cantón Chimbo, Ecuador, durante la pandemia COVID-19 en el período 2019-2020. La metodología: diseño no experimental, descriptivo, correlacional con corte transversal. La población de estudio 60 trabajadores de la Cooperativa. Los principales hallazgos, se encontraron cinco inferencias principalmente entre los síntomas fisiológicos, comportamiento social y lo intelectual-laboral con daños psicosomáticos, ansiedad e insomnio. Y percepción baja de salud en general por parte de los trabajadores. Las personas expuestas al estrés presentaron 10 veces más probabilidad de daño fisiológico. Como conclusión se determinó que dentro de los principales desencadenantes de problemas de salud es el estrés en los trabajadores de la cooperativa de estudio y que la tensión social y económica causada por la pandemia incrementó su exposición.

Palabras clave: estrés laboral; salud general; COVID-19.

Código de clasificación internacional: 3207.16 - Estrés.

Cómo citar este artículo:

Noroña, D., Quilumba, V., \& Vega, V. (2021). Estrés Laboral y Salud General en Trabajadores

Administrativos del área Bancaria. Revista Scientific, 6(21), 81-100, e-ISSN: 2542-2987.

Recuperado de: https://doi.org/10.29394/Scientific.issn.2542-2987.2021.6.21.4.81-100

Fecha de Recepción: 08-01-2021
Fecha de Aceptación:

14-07-2021
Fecha de Publicación: 05-08-2021 
OAI-PMH: http://www.indteca.com/ojs/index.php/Revista Scientific/oai

Artículo Original / Original Article

\title{
Occupational Stress and General Health in Administrative Workers of the Banking area
}

\begin{abstract}
In the different jobs, human beings are exposed to psychosocial risk factors, it is unavoidable and they are conditioned by the job. The appearance of COVID-19 increased the harmful effect on health. The objective of this research was to determine how work stress affects the general health of the administrative staff of the Association of savings and credits, San José, Chimbo canton, Ecuador, during the COVID-19 pandemic in the 2019-2020 period. The methodology: non-experimental, descriptive, correlational design with cross section. The study population 60 workers of the Cooperative. The main findings, five inferences were found mainly between physiological symptoms, social behavior and intellectual-work with psychosomatic damage, anxiety and insomnia. And low perception of health in general by workers. People exposed to stress were 10 times more likely to have physiological damage. As a conclusion, it was determined that among the main triggers of health problems is stress in the workers of the study cooperative and that the social and economic tension caused by the pandemic increased their exposure.
\end{abstract}

Keywords: work stress; general health; COVID-19. International classification code: 3207.16 - Stress.

How to cite this article:

Noroña, D., Quilumba, V., \& Vega, V. (2021). Occupational Stress and General Health in Administrative Workers of the Banking area. Revista Scientific, 6(21), 81-100, e-ISSN: 2542-2987. Recovered from: https://doi.org/10.29394/Scientific.issn.2542-2987.2021.6.21.4.81-100

Date Received: 08-01-2021
Date Acceptance: 14-07-2021
Date Publication: 05-08-2021 


\section{Introducción}

El crecimiento vertiginoso de la tecnología, los abundantes cambios laborales, las demandas de la vida cotidiana, lo que exige el trabajo en los últimos años y en la actualidad el desarrollo de la pandemia del COVID-19, han denotado consecuencias de estrés laboral, agotamiento emocional, ansiedad, depresión, entre otras, donde todo trabajador debido a la función que representa y enfrentan en su diario vivir en su ámbito laboral y más aún la exposición directa al COVID-19, tienen un alto riesgo de contagio, donde involucra a su familia, lo que incluso podría conllevarle a la muerte, y con ello al desarrollo de problemas conductuales, psicológicos y físicos que se pueden traducir en un estrés laboral, lo cual apunta en un impacto significativo en la salud mental de los trabajadores.

La salud mental incluye una amplia gama de actividades directas o indirectas, concernientes con el componente del bienestar mental, equivalente a la definición de salud que da la Organización Panamericana de la Salud junto con la Organización Mundial de la Salud (OPS-OMS, 2020a): poniendo de manifestó que no solo es la ausencia de las enfermedades, sino que el concepto involucra el desarrollo de otros factores como la autoestima, la grupalidad, el compromiso con el trabajo, las redes sociales de apoyo, el locus de control, entre otros.

En esa misma línea, Sandín (2003): expone que en términos sencillos se podría definir el estrés como un estado de tensión psíquica que se acompaña de cambios fisiológicos en donde las situaciones sociales pueden provocar altos niveles de estrés que, a su vez, afectan a la salud, a la calidad de vida. Para Rodríguez (2007), citado por Arias (2012a):

[...] El estrés tiene efectos perjudiciales para la salud, los especialistas distinguen dos tipos de estrés: uno positivo y otro negativo. El distrés, es un estado de tensión psíquica que se experimenta con malestar y el eustrés es un estado de tensión psíquica que favorece la activación necesaria para 
realizar diversas actividades. En ese sentido, los estudios de Selye permitieron determinar tres fases del estrés (pág. 526).

De lo anteriormente citado, se puede inferir que la tensión que genera ciertas tareas desafiantes en los puestos de trabajo, no siempre deviene en padecimientos para el trabajador. Cuando las actividades involucran mayor esfuerzo o empeño y no sobrepasan las competencias o recursos, se convierten en estímulos de aprendizaje que amplían el conocimiento, desarrollan las habilidades y perfeccionan las destrezas laborales. Para conseguir este resultado, también se necesitará que el trabajador tenga mayor libertad en decidir el modo de hacer las cosas, que encuentre por sí mismo nuevas alternativas de solución y planteamiento de problemas (creatividad), y que cuente con el tiempo y los recursos para un correcto desempeño.

Es por eso que el eustrés, está relacionado con la autoestima, la innovación, la conciencia social, la grupalidad y el proyecto de vida. Lamentablemente, estas características de los ambientes de trabajo saludables, son cada vez menos comunes cuando la empresa no invierte en capacitación, formación, reingeniería de procesos, adecuados procesos de selección de personal, estudios de tiempos y movimientos, entre otros.

Sin embargo, Arias (2012b): "el estrés se considera como un fenómeno mediador que actúa como gatillador de enfermedades como la diabetes mellitus, la artritis, la hipertensión, la cardiopatía coronaria, la gastritis, las úlceras pépticas, las alergias a la piel, el cáncer y otras" (pág. 526); como se ha manifestado, en momentos de emergencia o fuerte tensión emocional, el organismo a través de la segregación de hormonas activa el funcionamiento de los sistemas para una respuesta eficaz ante una intimidación externa. Este mecanismo evolutivo, permitió la supervivencia del hombre primitivo en una época en la cual eran frecuentes las amenazas de grandes depredadores.

El mecanismo bioquímico que libera la ACTH y la adrenalina en el 
torrente sanguíneo, no ha cambiado significativamente después de millones de años de evolución, pero los agentes disparadores de esta reacción bioquímica han sido sustituidos por los temores, por la ansiedad y las preocupaciones laborales.

Cuando el trabajador entra en la fase de alarma y resistencia del estrés, se liberan catecolaminas que preparan al cuerpo para el ataque o la huida con aumento de la frecuencia cardíaca e incremento en la irrigación sanguínea en músculos esqueléticos. Si el tiempo de exposición en el que estas substancias permanecen en el cuerpo es habitual y reiterado, compromete la actividad de los sistemas sanguíneo, digestivo e inmunológico. Es por lo que la mitigación del estrés laboral debe ser una preocupación empresarial de salud pública, ya que contribuye indirectamente en la lucha contra padecimientos tales como la diabetes, la hipertensión y el cáncer. De acuerdo con Arias, Herrero, Cabrera, Chibás y García (2020):

El siglo XXI se ha caracterizado desde sus inicios por una problemática de salud que ha afectado al mundo y Cuba no ha podido escapar de esta situación, que va desde un incremento de la resistencia microbiana, hasta la aparición de nuevas enfermedades infecciosas emergentes y reemergentes, como la de la COVID-19 a finales del pasado año (pág. 3).

La OPS-OMS (2020b): declaran emergencia sanitaria a nivel mundial, debido a la magnitud por contagio de COVID-19, causante del síndrome respiratorio agudo severo (SARS-CoV-2). Según Accini, et al. (2020): se refirió a las autoridades gubernamentales con las adecuadas recomendaciones para el afrontamiento de la nueva pandemia, en los distintos establecimientos de atención.

Acorde con el Instituto Ecuatoriano de Seguridad Social (IESS, 1986): en virtud de la normativa legal vigente del Ecuador, en el Decreto Ejecutivo 2393, se establece que es un deber por parte de los empleadores asegurar sitios saludables con base a gestión de los factores de riesgo (entre ellos el 
estrés laboral), para reducir la probabilidad de ocurrencia y sus consecuencias en enfermedades y accidentes laborales. Así también el Acuerdo Ministerial nro. MDT-2015-0088 del Ministerio de Trabajo (MDT, 2015): dispone de la evaluación periódica de los agentes psicosociales para propiciar planes de mitigación que aseguren lugares de trabajo sanos. Es el espíritu de amparo tutelar de estas dos leyes lo que justifica la estimación del estrés en todo centro de trabajo, puesto que, como se ha mencionado, es un compromiso ineludible de los empleadores.

La Cooperativa de Ahorro y Crédito San José, fue creada en el año 1964, por la Iglesia local como instrumento de lucha contra la pobreza, cuenta con agencias en las provincias de Bolívar, Los Ríos, Tungurahua, Pichincha. El personal administrativo de la Cooperativa de Ahorro y Crédito San José no está exento de sufrir la tensión y problemas relacionados con el estrés por lo que la investigación aportará en el estudio de cómo el estrés laboral ha afectado la salud general del personal administrativo en la pandemia.

Considerando que el estrés es un problema de salud pública y que afecta el desempeño y calidad de vida de las personas, es necesario este tipo de estudios que permite tener la percepción real de la situación que presenta el personal administrativo de la Cooperativa de Ahorro y Crédito San José, cantón Chimbo.

En tal virtud, el objetivo del estudio fue determinar el grado de afectación que el estrés laboral ejerce en la salud general del personal de esta empresa durante la pandemia COVID-19 en el período 2019-2020. Para tal propósito, se midió el nivel de exposición de estrés laboral y se caracterizó la percepción de salud de los trabajadores.

\section{Metodología}

La presente investigación se desarrolló bajo un diseño no experimental, descriptivo, correlacional con corte transversal, donde se detallará el 
comportamiento del estrés en el personal de la Cooperativa y de qué manera este repercute en la salud de los trabajadores. La primera variable es la exposición de estrés laboral y la segunda es la caracterización de la autopercepción de la salud del personal.

La población inicialmente fue de 80 trabajadores, pero al aplicar los criterios de selección se confirmó una población final de estudio de 60 informantes. Los criterios de inclusión fueron los siguientes: personas mayores de 18 años, con tipo de contrato eventual, ocasional, e indefinido, que laboraban bajo relación de dependencia en el área administrativa y que firmaron el consentimiento informado. Se excluyeron a quienes al momento de la recolección de información se encontraban en vacaciones, baja médica o proceso de desvinculación. Finalmente, se eliminaron las encuestas entregadas de manera incompleta.

Con relación a los instrumentos empleados, se utilizó el Cuestionario para la Evaluación del Estrés - Tercera versión del Ministerio de la Protección Social (MPS, 2010): que se encuentra conformado de 31 preguntas y su valoración de respuesta es a través de una escala de Likert, la forma de respuesta es en función de la frecuencia de ocurrencia de los diferentes malestares presentados en los últimos tres meses.

La finalidad es la de evaluar la presencia de reacciones de estrés, este instrumento se divide en cuatro categorías: fisiológicos $(1,2,3,4,5,6,7,8)$, comportamiento social $(9,10,11,12)$, intelectuales y laborales $(13,14,15,16$, $17,18,19,20,21,22)$, y psicoemocionales $(23,24,25,26,27,28,29,30,31)$. Y para la variable de salud se utilizó el cuestionario general de salud de Goldberg, considerando subescalas: A. (síntomas somáticos 1-7), B. (ansiedad e insomnio 8-14), C. (disfunción social 15-21), D. (depresión grave 22-30). Los resultados se califican de 0 a 4 puntos: ausencia de psicopatología, 5 a 6 puntos: sospecha de psicopatología subumbral, 7 a 12 puntos: indicativos de presencia de psicopatología. En cuanto al índice de 
fiabilidad, el alfa de Cronbach es de 0,95. Los resultados finales del instrumento se presentan en cuatro categorías, estrés muy bajo, bajo, medio, alto y muy alto.

El segundo instrumento fue el Cuestionario de Salud General de 30 ítems (GHQ-30). Instrumento auto administrado que mide la salud mental, con una validez interna de 0,89 y compuesto de 30 preguntas en 4 dimensiones: síntomas psicosomáticos, ansiedad e insomnio, disfunción social y depresión. Las respuestas se encuentran en escala de Likert con opciones del 1 al 4 . La forma de calificación conlleva a que se sumen las veces en que el participante señaló las opciones 3 y 4 , y si su suma es mayor a 7 representa casos o perturbaciones en la salud mental.

Para las pruebas estadísticas, se realizaron 3 análisis: descriptivo, inferencial y de asociación. En el primero de ellos, se calcularon las frecuencias y porcentajes de las variables sociodemográficas, del estrés y de la salud mental manejando las escalas de calificación de los instrumentos anteriormente señaladas. La segunda prueba, conllevó el cálculo de Chicuadrado $X^{2}$ con un nivel de significancia del 0,05. En el tercer test, finalmente se calculó el Odds Ratio (OR) o razón de momios para comprobar la probabilidad de ocurrencia.

Se emplearon consideraciones éticas establecidas en el consentimiento informado. En todo momento se socializó del alcance y propósito del estudio a los participantes haciendo hincapié de la confidencialidad de los datos y que sus resultados servirían para fortalecimiento de las condiciones actuales de trabajo.

\section{Resultados}

\subsection{Datos Generales}

En las variables sociodemográficas, la participación del género masculino fue mayor con 32 en relación con 28 del femenino, el rango de edad 
de mayor frecuencia fue de 31 a 40 años que se presentan en 40 personas de la población de la agencia bancaria. Para el estado civil, el grupo de los casados es mayor con 36 , seguido los solteros con 19, la unión libre con 4 y tan solo 1 individuo divorciado.

Tabla 1. Factores sociodemográficos (cuestionario aplicado a los trabajadores).

\begin{tabular}{|c|c|c|c|}
\hline Edad/Género & Femenino & Masculino & Total \\
\hline $20-30$ & 1 & 7 & 8 \\
\hline $31-40$ & 23 & 17 & 40 \\
\hline $41-50$ & 4 & 7 & 11 \\
\hline Más 60 & 0 & 1 & 1 \\
\hline Total \% & $28(46,67 \%)$ & $32(53,33 \%)$ & $60(100,00 \%)$ \\
\hline Estado/Género & Femenino & Masculino & Total \\
\hline Casado & 22 & 14 & 36 \\
\hline Divorciado & 0 & 1 & 1 \\
\hline Soltero & 5 & 14 & 19 \\
\hline Unión libre & 1 & 3 & 4 \\
\hline Total \% & $28(46,67 \%)$ & $32(53,33 \%)$ & $60(100,00 \%)$ \\
\hline Nivel de Instrucción & Femenino & Masculino & Total \\
\hline Cuarto nivel & 11 & 8 & 19 \\
\hline Secundaria & 0 & 4 & 4 \\
\hline Tercer nivel & 17 & 20 & 37 \\
\hline Total \% & $28(46,67 \%)$ & $32(53,33 \%)$ & $60(100,00 \%)$ \\
\hline
\end{tabular}

Fuente: Los Autores (2021).

Como se puede observar en la tabla 1, se trata de una población joven, en su mayoría casados y con estudios universitarios ya concluidos. Existen diferencias entre género con el estado civil, las mujeres registran mayor frecuencia para el grupo casado con 22, y el hombre con el mayor número es para soltero con 14. En general el grupo muestra paridad con respecto al nivel de instrucción, aunque se puede indicar que 4 de los trabajadores que no cuentan con títulos universitarios son hombres de segundaria y que 11 de las mujeres presentan un mayor índice de estudios de cuarto nivel. 
Tabla 2. Puesto de trabajo / Carga horaria (cuestionario aplicado a los trabajadores).

\begin{tabular}{|l|c|c|c|}
\hline $\begin{array}{c}\text { Puesto de Trabajo I } \\
\text { Carga Horario }\end{array}$ & $\begin{array}{c}\text { No realiza } \\
\text { horas extra }\end{array}$ & $\begin{array}{c}\text { Realiza horas } \\
\text { extra }\end{array}$ & Total \\
\hline Analista & 1 & 7 & 8 \\
\hline Asesor Jurídico & 1 & 0 & 1 \\
\hline Asistente & 11 & 11 & 22 \\
\hline Auditora & 1 & 0 & 1 \\
\hline Chófer mensajero & 0 & 1 & 1 \\
\hline Cobranzas & 1 & 0 & 1 \\
\hline Conserje Mensajero & 0 & 2 & 2 \\
\hline Conserje mensajero & 0 & 1 & 1 \\
\hline Contador & 0 & 2 & 2 \\
\hline Coordinador & 2 & 6 & 8 \\
\hline Jefe & 4 & 9 & 13 \\
\hline Total \% & $21(35,00 \%)$ & $39(65,00 \%)$ & $60(100,00 \%)$ \\
\hline
\end{tabular}

Fuente: Los Autores (2021).

En la tabla 2, los cargos en los que cuentan con mayor personal son los asistentes con 22, jefe 13 y para el analistas y coordinadores 8 . Por otro lado, para complementar el análisis de estrés laboral, se indagó a los participantes sobre si realizan horas extras a la semana. Cabe recalcar que, los pagos de las horas extras no son contabilizadas en la remuneración mensual del trabajador y que el tiempo que destinan los trabajadores corre por su propia cuenta.

El orden en el que se suman las horas extras en la organización corresponde primero a los jefes, seguido de los analistas, los asistentes y los coordinadores. Las personas responsables de la dirección de otras y los asistentes, quienes laboran en las cajas de la agencia bancaria, emplean diariamente más de las ocho horas de su jornada en el cumplimiento de sus deberes, funciones y responsabilidades. 


\subsection{Exposición al estrés laboral}

Para la variable de estrés laboral, el cuestionario utilizado presenta 4 dimensiones que son factores fisiológicos (FF), comportamiento social (CS), intelectual y laboral (IL), y psicoemocionales (PE). En los resultados, el nivel de riesgo que prevalece es el estrés medio, y la categoría con mayor afectación, corresponde a factores fisiológicos, seguido de los intelectuales laborales.

Teniendo en cuenta que el estrés medio significa la existencia de señales notorias de afectación por estrés laboral y que el estrés alto simboliza la confirmación de elevado deterioro por su exposición; se puede deducir que su suma representa la totalidad de las personas expuestas a este factor psicosocial.

Tabla 3. Resultado de estrés (cuestionario aplicado a los trabajadores).

Fuente: Los Autores (2021).

\begin{tabular}{|l|c|c|c|}
\hline \multicolumn{1}{|c|}{ DIMENSIONES } & ALTO & MEDIO & BAJO \\
\hline Síntomas fisiológicos & $4(6,7 \%)$ & $36(60,0 \%)$ & $20(33,3 \%)$ \\
\hline Comportamiento social & $2(3,3 \%)$ & $24(40,0 \%)$ & $34(56,7 \%)$ \\
\hline Intelectuales y laborales & $4(6,7 \%)$ & $23(38,3 \%)$ & $33(55,0 \%)$ \\
\hline Psicoemocionales & $2(3,3 \%)$ & $23(38,3 \%)$ & $35(58,3 \%)$ \\
\hline \multicolumn{4}{|c}{$\mathbf{X = 6 0}$} \\
\hline
\end{tabular}

En este sentido, como se expone en la tabla 3, la dimensión del estrés síntomas fisiológicos tiene $4(6,7 \%)$ personas en categoría alta y $36(60,0 \%)$ en medio, su suma indica que son $40(66,7 \%)$ personas, que presentan dolencias físicas de moderadas a graves con sintomatología de dolores de cuello, espalda y tensión muscular. Los factores intelectuales y laborales tienen en alto al $4(6,7 \%)$ de los informantes y en medio a $23(38,3 \%)$ sujetos dando una suma que representa el $27(45 \%)$ de la población padeciente de sobrecarga de trabajo, dificultad para concentrarse, cansancio, tedio 0 desgano.

En los problemas de comportamiento social en los cuales hay $2(3,3 \%)$ 
trabajadores en alto y $24(40,0 \%)$ en medio de $26(43,3 \%)$ sujetos que presentan incapacidad para socializar, trabajar en equipo y obtener retroalimentación efectiva. Finalmente, para la dimensión de problemas psicoemocionales, son $2(3,3 \%)$ personas en alto y $23(38,3 \%)$ en medio que da como resultado a 25 trabajadores que representa el $41,6 \%$ de exposición en donde se caracterizó por sentimientos de angustia, preocupación, tristeza, necesidad de consumo de bebidas alcohólicas.

\subsection{Resultados de la autopercepción de salud}

Al aplicar el cuestionario general de salud de Goldberg, para la identificación de la presencia de problemas de salud en los trabajadores que fueron parte de la investigación, se obtuvo resultados que caracterizaron la percepción de la depresión, la disfunción social, la ansiedad e insomnio y de los síntomas asintomáticos de los trabajadores de la Cooperativa.

Tabla 4. Resultado del cuestionario de salud mental

\begin{tabular}{|l|c|c|c|}
\hline Dimensiones & Presencia & Sospecha & Ausencia \\
\hline Depresión grave & $2(3,3 \%)$ & $0(0,0 \%)$ & $58(96,7 \%)$ \\
\hline Disfunción social & $11(18,3 \%)$ & $0(0,0 \%)$ & $49(81,7 \%)$ \\
\hline Ansiedad e insomnio & $12(20,0 \%)$ & $1(1,7 \%)$ & $47(78,3 \%)$ \\
\hline Síntomas Psicosomáticos & $11(18,3 \%)$ & $0(0,0 \%)$ & $49(81,7 \%)$ \\
\hline
\end{tabular}

Fuente: Los Autores (2021).

Como se expone en la tabla 4, las dimensiones del cuestionario de Goldberg, confirman 11 casos (18,3\%) con síntomas psicosomáticos en donde los informantes padecen dolencias físicas inespecíficas, al igual que las personas con disfunción social que impide la adaptación correcta a las funciones de sus actividades en lo referente a la comunicación y el relacionamiento, 2 personas $(3,3 \%)$ con depresión y $12(20 \%)$ con ansiedad e insomnio. 


\subsection{Relación Estrés y problemas de salud}

Para establecer la relación existente entre las variables de estudio se utilizó prueba de Chi-cuadrado $\mathrm{X}^{2}$ entre los niveles de estrés sobre las categorías de salud presentadas, en donde se observa que según los resultados cinco relaciones estadísticamente significativas (significancia) al ser menor a $p=0.05$. Esta información determina que el nivel de estrés incide significativamente en la presencia de enfermedades en los trabajadores participantes de la investigación.

Tabla 5. Inferencias entre estrés con salud, cálculo de $X^{2}$.

\begin{tabular}{|l|c|c|c|c|}
\hline \multicolumn{1}{|c|}{ INFERENCIAS } & $\begin{array}{c}\text { Síntomas } \\
\text { fisiológicos }\end{array}$ & $\begin{array}{c}\text { Comportamiento } \\
\text { social }\end{array}$ & $\begin{array}{c}\text { Intelectuales } \\
\text { y laborales }\end{array}$ & Psicoemocionales \\
\hline Daños psicosomáticos & $0,001^{*}$ & $0,044^{*}$ & $0,032^{*}$ & 0,376 \\
\hline Disfunción social & 0,199 & 0,655 & 0,234 & 0,234 \\
\hline Depresión grave & 0,824 & 0,841 & 0,164 & 0,822 \\
\hline Ansiedad e insomnio & $0,008^{*}$ & $\begin{array}{c}0,19 \\
\left(^{*}\right) \text { nivel de significación del } \mathbf{0 , 0 5}\end{array}$ & $0,02^{*}$ & 0,28 \\
\hline
\end{tabular}

Fuente: Los Autores (2021).

Cómo se presenta en la tabla 5 , se confirmaron cinco relaciones estadísticamente significativas. Los daños psicosomáticos en la salud están relacionados con los síntomas fisiológicos, el comportamiento social y los factores intelectuales-laborales del estrés laboral. Por otra parte, la ansiedad y el insomnio se encuentra inferenciado con los síntomas fisiológicos y los intelectuales laborales.

\subsection{Asociación entre estrés y salud}

Para la identificación del nivel de relación de las variables de estudio se aplicó la razón de momios u Odds Ratio (OR), para el cual se utilizaron las cinco inferencias encontradas en la prueba del chi-cuadrado. El valor OR es significativo cuando se cumplen tres condiciones: el valor de $\mathrm{X} 2$ debe ser menor al nivel de significancia de 0,05, el intervalo de confianza no debe contener a la unidad y el valor OR debe ser mayor a la unidad. 
Tabla 6. Cálculo del OR en las relaciones estadísticamente significativas.

\begin{tabular}{|l|c|c|c|}
\hline \multicolumn{1}{|c|}{ INFERENCIAS } & \multirow{2}{*}{ OR } & \multicolumn{2}{c|}{ INTERVALO DE CONFIANZA } \\
\cline { 3 - 5 } & & Límite Inferior & Límite Superior \\
\hline Síntomas fisiológicos *Daños psicosomáticos & 10,1 & 1,142 & 28,779 \\
\hline Comportamiento social *Daños psicosomáticos & 1,95 & 0,895 & 29,241 \\
\hline Intelectuales y laborales *Daños psicosomáticos & 1,78 & 0,479 & 431,471 \\
\hline Síntomas fisiológicos *Ansiedad e insomnio & 4,335 & 0,479 & 431,471 \\
\hline Intelectuales y laborales *Ansiedad e insomnio & 16,3 & 0,479 & 431,471 \\
\hline
\end{tabular}

Fuente: Los Autores (2021).

En la tabla 6, de las cinco inferencias registradas por el $X^{2}$, tan solo existió una probabilidad de ocurrencia para síntomas fisiológicos y daños psicosomáticos. Las personas que por estrés registran dolencias de dolor en el cuello, espalda y tensión muscular, trastornos de sueño, problemas gastrointestinales y cambios fuertes de apetito, presentan hasta 10 veces más probabilidad de sufrir dolencias físicas reales incapacitantes. El resto de las inferencias, si bien obtuvieron un OR por encima de 2, su intervalo de confianza contuvo a la unidad, por lo que no llegó a ser representativo.

EI OR o razón de momios, es una prueba estadística que evalúa la probabilidad de ocurrencia de una variable dependiente con base a la presencia de su factor relacionado. Para su cálculo, es preciso desarrollar una tabla de $2 \times 2$ en la cual se sitúan en posición de columnas las personas que presentaron daños psicosomáticos y a continuación quienes no presentaron esta perturbación. En filas, se disponen de los factores con relación a los expuestos a síntomas fisiológicos, comportamiento y factores sociales intelectuales. La tabla 7, explica el cálculo del estadístico.

Tabla 7. Explicación del cálculo del Odds Ratio.

\begin{tabular}{|c|c|c|c|}
\cline { 2 - 4 } \multicolumn{1}{c|}{} & Presenta daño & No presenta daño & Total \\
\hline Estuvieron expuestos & $\mathrm{a}$ & $\mathrm{b}$ & $\mathrm{a}+\mathrm{b}$ \\
\hline No estuvieron expuestos & $\mathrm{c}$ & $\mathrm{d}$ & $\mathrm{c}+\mathrm{d}$ \\
\hline Total & $\mathrm{a}+\mathrm{b}$ & $\mathrm{b}+\mathrm{d}$ & $\mathrm{a}+\mathrm{b}+\mathrm{c}+\mathrm{d}$ \\
\hline
\end{tabular}

Fuente: Los Autores (2021). 
Finalmente se procede a realizar la siguiente operación:

$$
O R=\frac{a / b}{c / d}=\frac{a * d}{c * d}
$$

Con el avance de los paquetes estadísticos, se optó por el cálculo directo sin necesidad de computar los resultados parciales. En este estudio se utilizó el programa estadístico informático SPSS versión 23 para su deducción.

\section{Conclusiones}

La edad promedio del personal administrativo de la Cooperativa de ahorro y crédito San José del Cantón de Chimbo que ha sido investigado, es de 36 años, de estos el 28 (46,67\%) son mujeres y el 32 (53,33\%) hombres, la mayor parte son de estado civil 36 son casados y 19 solteros; en cuanto al nivel de instrucción 37 tienen tercer nivel y 19 cuarto nivel.

En relación con los niveles de estrés el personal administrativo de la Cooperativa de ahorro y crédito San José del Cantón de Chimbo, presenta un nivel de riesgo medio es decir este problema de salud se encuentra latente, las categorías en donde mayor problema se refleja son los factores fisiológicos y factores intelectuales y laborales. Los síntomas característicos dentro de la primera categoría son dolor en el cuello, espalda y tensión muscular, además trastornos de sueño durante todo el día, problemas gastrointestinales, úlcera péptica, acidez, problemas digestivos o del colon, y, cambios fuertes de apetito; mientras que de la segunda se destaca el sentimiento de sobrecarga de trabajo, dificultad para concentrase y cansancio, tedio o desgano.

Los problemas de salud prevalentes en el personal administrativo de la Cooperativa debido a la presencia de estrés se destacan en las categorías de disfunción social, ansiedad e insomnio y síntomas asintomáticos, los signos que presentaron miedo y sensación de ser vigilado; así como intranquilidad y dificultad de conciliar el sueño, así como pesadez y sensación que va a estallar su cabeza. 
La dependencia entre la presencia de estrés y problemas de salud que se determinó en esta investigación fue positiva debido a que existió una correlación entre las variables, lo cual significa que los problemas de salud que se encontraron tienen relación directa con el estrés, y que a futuro se podrán prevenir si se actúa de forma inmediata.

\section{Referencias}

Accini, J., Beltrán, N., Nieto, V., Ramos, E., Pizarro, C., ... Torres, V. (2020). Declaración de consenso en medicina crítica para la atención multidisciplinaria del paciente con sospecha o confirmación diagnóstica de COVID-19. Acta Colombiana de Cuidado Intensivo, 20(4), 287-333, e-ISSN: 0122-7262. Recuperado de:

https://doi.org/10.1016/j.acci.2020.04.003

Arias, W. (2012a,b). Estrés laboral en trabajadores desde el enfoque de los sucesos vitales. Revista Cubana de Salud Pública, 38(4), 525-535, e-ISSN: 1561-3127. Recuperado de:

http://www.revsaludpublica.sld.cu/index.php/spu/article/view/180

Arias, Y., Herrero, Y., Cabrera, Y., Chibás, D., \& García, Y. (2020). Manifestaciones psicológicas frente a la situación epidemiológica causada por la COVID-19. Revista Habanera de Ciencias Médicas, 19(supl.), 1-13, e-ISSN: 1729-519X. Recuperado de:

http://www.revhabanera.sld.cu/index.php/rhab/article/view/3350

IESS (1986). Reglamento de seguridad y salud de los trabajadores y mejoramiento del medio ambiente de trabajo. Decreto Ejecutivo 2393, Registro Oficial 565, Última modificación: 21-feb-2003. Ecuador: Instituto Ecuatoriano de Seguridad Social.

MDT (2015). Acuerdo Ministerial N. ${ }^{\circ}$ MDT-2015-0088. San Francisco de Quito, Ecuador: Ministerio del Trabajo.

MPS (2010). Batería de instrumentos para la evaluación de factores de 
riesgo psicosocial. ISBN: 978-958-8361-93-2. Bogotá, Colombia: Ministerio de la Protección Social.

OPS-OMS (2020a,b). Consideraciones psicosociales y de salud mental durante el brote de COVID-19. Washington, Estados Unidos: Organización Panamericana de la Salud; Organización Mundial de la Salud.

Sandín, B. (2003). El estrés: un análisis basado en el papel de los factores sociales. International Journal of Clinical and Health Psychology, 3(1), 141-157, e-ISSN: 1697-2600. Recuperado de:

https://www.redalyc.org/articulo.oa?id=33730109 


\section{Darwin Raúl Noroña Salcedo}

e-mail: darwin norona@yahoo.com

Nacido en Quito, Ecuador, el 13 de marzo del año 1983.

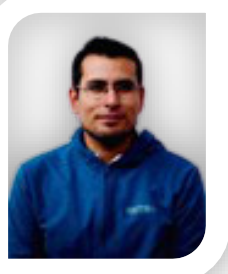

$\mathrm{PhD}$. en Ciencias de la Salud Ocupacional por la Universidad de Guadalajara (UDG), México; además de Magister en Seguridad Laboral por la Universidad Central del Ecuador (UCE); Profesional en el área de gestión del talento humano; cambio de cultura organizacional; violencia de género; discriminación e interculturalidad y en investigación en seguridad y salud ocupacional; con aptitudes de liderazgo, investigación, desarrollo y ejecución de proyectos técnicos; especial énfasis en dirección de equipos de trabajo, consultorías profesionales y en docencia universitaria. 


\section{Valeria Alejandra Quilumba Vallejo}

e-mail: conejavqv@gmail.com

Nacida en Ambato, Ecuador, el 13 de marzo del año 1986.

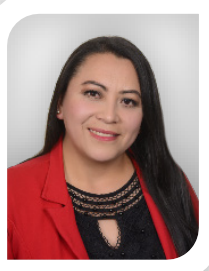

Magister en Salud Ocupacional por la Universidad Regional Autónoma de Los Andes (UNIANDES); poseo un espíritu humanista y de servicio, dispuesta a trabajar en equipo, con disposición de superación y actualización permanente; transmisora de conocimientos; experiencias a pacientes, familiares y compañeros; la premisa de mis acciones son de responsabilidad social, actuando conforme la ética médica; en la actualidad desempeño mis funciones en el Centro Salud de Santa Rosa Tipo B en atención primaria de salud y servicio en áreas comunitarias, mediante trabajo intramural y extramural. 


\section{Vega Falcón Vladimir \\ e-mail: vega.vladimir@gmail.com}

Nacido en Matanzas, Cuba, el 18 de agosto del año

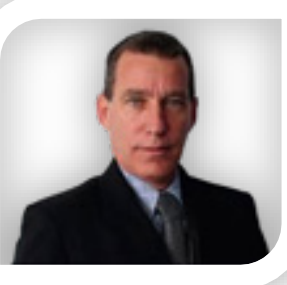
1964. PhD en Ciencias Económicas; Categoría Docente Principal de Profesor Titular (Catedrático); actualmente Docente Investigador con dedicación a Tiempo Completo, Titular Principal Nivel de Escalafón 3 de la Universidad Regional Autónoma de los Andes (UNIANDES), Ecuador; he colaborado sistemáticamente como docente de pregrado y postgrado en universidades de varios países, abordando diversas temáticas, entre las que se encuentran: Contabilidad de Costos, Contabilidad de Gestión Avanzada, Planeación Estratégica y Cuadro de Mando Integral, Herramientas gerenciales para toma de decisiones, Capital Intelectual, Gestión Turística, Metodología de la Investigación, Redacción de artículos científicos.

\footnotetext{
El contenido de este manuscrito se difunde bajo una Licencia de Creative Commons Reconocimiento-
} NoComercial-Compartirlgual 4.0 Internacional 